\title{
MAIZ: A Web Mashup Application for Information Services to CitiZens During Disaster Situations
}

\author{
Pablo Pancardo \\ DAIS \\ Juarez Autonomous University of Tabasco (UJAT) \\ Cunduacan, Tabasco, Mexico.
}

\author{
Miguel A. Wister \\ DAIS \\ Juarez Autonomous University of Tabasco (UJAT) \\ Cunduacan, Tabasco, Mexico.
}

\author{
Francisco Acosta \\ DAIS \\ Juarez Autonomous University of Tabasco (UJAT) \\ Cunduacan, Tabasco, Mexico.
}

\begin{abstract}
In this paper is presented an information system based on Web Mashup application for offering various information services in disaster situation (floods). This system will allow damaged citizens wearing mobile devices to discover services such as: emergency shelters and housing, flooded neighborhood, collection centers, river level, red cross, flood-damaged roads, jammed roads, and so on; these citizens could be suffering from crisis due to flood damage. We consider the possibility of designing an information system based on Web Mashup application to make use of several contents retrieved from external data sources and maps to create services for guiding people who cannot be found by their families, rescuing injured people, helping people who need to be rescued from their inundated homes, moving people to hospitals or emergency shelters, and other available services near their current location. The aim of this work is to propose a framework based on existing services to able to support a complete set of services to alert people in risk situation, help and rescue people affected by a natural disaster. This is the main difference with related works such as those been proposed in the context of Katrina flood in New Orleans.
\end{abstract}

\section{General Terms:}

Web Mashup application, Disaster situations

\section{Keywords:}

Ambient Intelligence, Wireless Networking, Web Services

\section{INTRODUCTION}

After occurring floods, power outages and damages of electrical systems could be found in a city. It could also result in existing networking infrastructure being damaged or becoming unreliable and communication means for people cannot be used in the disaster area. Problems like these occurred during Hurricane Katrina were dealt with some works [1] [2]. It is commonly known that cellular networks are often broken down due to many calls for confirming safety of inhabitants from outside disaster area. Traditional wireless networks are unable to offer a solution, as they become unstable. Therefore, it is required to design disaster tolerant architecture [3] [4] and prepare emergency network system, which can be quickly reconstructed to recover from the network failure [5].
Originally, this work is related to deploy wireless ad-hoc and mesh networks to support services for relief and rescue in disaster situations. In Tabasco floods occur annually and we are very interested in this case study because it is very helpful to people. It is typical when a city is flooded by the overflowing of rivers, an important part of the population must be evacuated and taken to emergency shelters and housing, hospitals, and higher lands. During a disaster is required medicines, drinking water, food, police, fire brigades, helicopters, boats, and many other services.

There is a previous phase of this work related to Wireless Sensor Networks (WSN) placed on rivers near the city, these WSN send alert messages to the people when river levels rise and allow time for these people to evacuate or make decision. This system monitors river levels using sensors to alert population living in flood-prone areas and allows for intercommunication among mobile devices that collect information from users via several wireless communication modes. Other services are considered for urban flooding rescue scenario. The whole system includes three wireless networks such as a WSN to measure the water levels in rivers, a Mobile Ad Hoc Network (MANET) to communicate rescuers and victims, and a Wireless Mesh Network (WSN) to connect the MANET with government agencies and civil protection.

What may happen in the city after rivers overflow; we intend to design an information system running on a MANET that provides support to users during and after an emergency occurs, so that these users will be surrounded by an infrastructure available even with the damage that could have caused the disaster. Besides some ideas of Ambient Intelligence (AmI) to the interaction between users and the stage of flooding. Also, we are using concepts related to context and location awareness for helping to users in these tasks.

In disaster situations, users can dial their mobile telephones, but if the lines are saturated or collapsed, or emergency telephone number is unknown or electrical power is not available or users (older people) do not know how to use cell telephones, and a long list adverse events, the requests for help will be too difficult. Applications previously installed on the user's mobile devices could be helpful.

As mentioned in above paragraph, we have already been previously published [6] [7], other wide range of applications for both MANET and WSN technologies centered to disaster situa- 
tions. [8] [9] [10] [11] [12]. Moreover, using WSN, MANET and WMN we are considering to design an information system based on Web Mashup application to combine maps and data for guiding users to emergency shelters, collection center, information about closed roads and other available services near their current position.

The remainder of the paper is organized as follows: Section 2 includes some related works. Section 3 describes Mashup technology. In section 4 is described the method to build a Web Mashup application and its application related to this study case. Section 5 gives the description of services contained in the Web Mashup application, both civil protection offices services and citizens services. Finally, section 6 closes giving preliminary results and conclusions.

\section{RELATED WORK}

There are many projects that their applications are focused on disasters by floods. One of them is ALERT [13] (ALERT is an acronym for Automated Local Evaluation in Real Time), ALERT is a system for flood detection, which uses remote sensors to send environmental data to a computer system. Various kind of sensors deployed in the ALERT system is rainfall, water level and weather sensors. These sensors give information to the centralized database system.

A work is found in [14] it is a landslide detection system using a WSN. This system has enabled a more convenient early warning system and provides a system able to learn about the phenomena of natural disasters.

Other works have proposed solutions related to AmI centered in healthcare, social wellness, ambient assisted living (AAL), home assistant. In [15] is presented a vision of how AmI environments may be used in future to support emergency services during first response.

\section{MASHUP TECHNOLOGY}

Currently, most Web applications work getting information contained in Web servers where are hosted the application. Actually, operations are requested to servers that offer only what is contained itself; but, do not provide an integrated interface to display information from different applications on different Web servers, and even less access to Web services available in Internet, which is a classic pattern of Web 1.0.

Web 2.0 consists of the transition on Internet from traditional Web applications to Web applications user-centered, is a set of services that exceed the limitations. It is talking about integrated interfaces, which on a single page it may allow available features and interfaces to other Web sites, so do not be confused with a page with links (links) to other sites, the concept goes further.

After arriving Web 2.0 in 2005, it emerged the concept of Mashup (Web application hybrid). It is characterized by the usage of the resources from other Web sites, combining to provide an integrated experience. These resources are provided by third parties called Web Services, that is a generic term that describes a collection of different services or functionality that Web sites available to others, these services are built based on industry standard protocols that facilitate interoperability between applications.

Therefore, Mashups are Web applications that combine data and content from more than one source into an aggregated user experience. They are the next stage in the evolution of Web 2.0 applications.

A Mashup is a Web application that combines content and data, typically from third parties via public services APIs, into an aggregated user experience. Sources of content and data may be from Web feeds (RSS or Atom), Web services, and screen scraping. To date mapping, photo sharing, and video sharing services lead the pack of applications well-suited to Mashups. Also, rich browser-based Mashup editors are coming on the scene that merge many services including rich UI component.

There exist many advantages in this type of hybrid applications, to list one positive features, e.g., a system engineer do not need much knowledge to develop a lot of software, since many components are already programmed using specific functions that can be carried out, it is necessary only to know how to use the resources that will be supported.

On the other hand, one factor contributing to the success of Web services is being developed using Internet standards such as XML and HTTP, which allows systems to be able to analyze text and communicate through a transport protocol for Internet to a Web service.

\section{METHOD TO BUILD A MASHUP}

The following is a general method for creating a Web Mashup application. This method is defined from the examples shown by different software companies on Internet and can be seen in Figure 1.

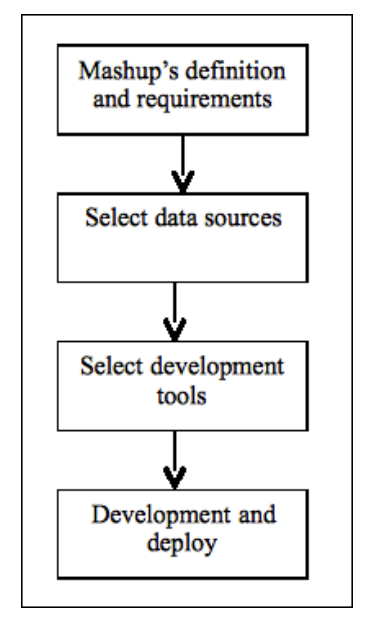

Fig. 1. Method to build a Web Mashup application.

\subsection{Mashup definition and requirements}

MAIZ has been defined as a Web Mashup application that serves as a liaison between civil protection authorities and population of the area where occurs a disaster, specially, those people affected by floods. Thus defined information services are intended to fill the gaps in information support required by people involved in the incident. MAIZ's case study and scenario is Villahermosa city, in Tabasco, Mexico, where, as mentioned earlier, in recent years have caused flooding on a recurring basis.

In this case of study and based on experience about information requirements during last floods in Tabasco, it is established that applications functional requirements are: point of interest such as flooded neighborhood, river levels, closed roads, emergency shelters, Red Cross, collection centers, medical care, and so forth; Web search about news related to flooding, chat (conversation), and, RSS services. The main features of MAIZ, both for administrators (civil protection authorities) and users (citizens) of the system is shown in the UML use case diagram illustrated in Figure 2. 


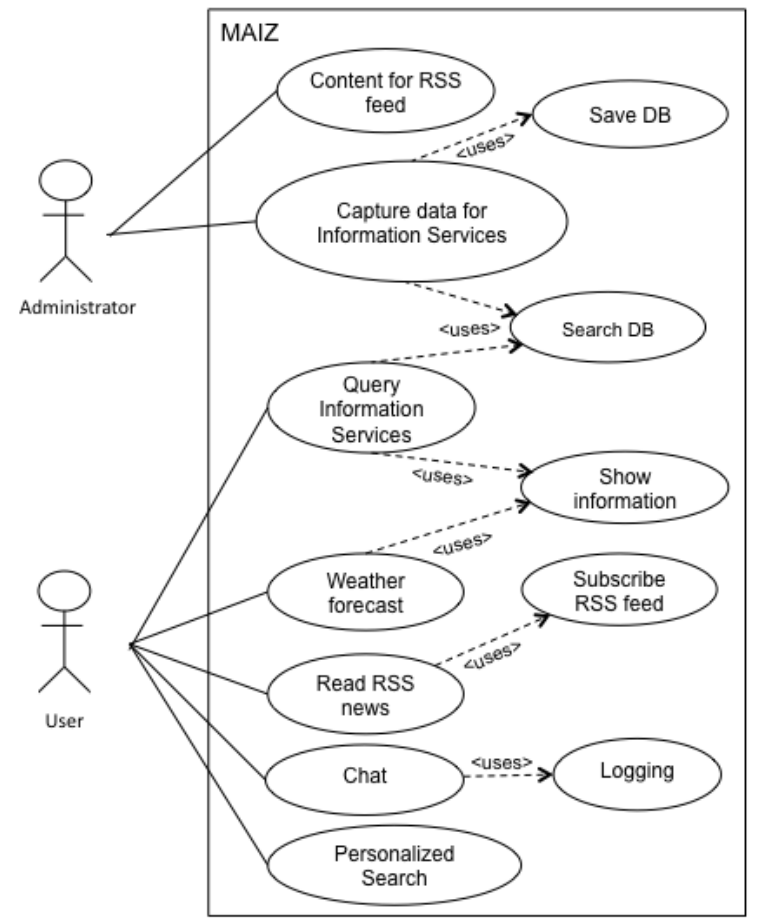

Fig. 2. MAIZ's UML Use Case Diagram.

\subsection{Select data sources}

For information services to those people affected by floods, which need use maps of the affected area to show the place (address) of the service, using Google Maps API. This API is a set of services that allow to include maps, geocoding data and other Google content in Web pages or applications. So, it can be carried out in a simple way and free Google maps, transforming them into an application that provides certain functionality and include it in any public free website.

Google Maps API allows client applications to view, save and update the data map in the Google API feeds format through a functions data model (placemarks, lines and shapes) and maps (collections of functions).

Google Maps services were chosen to be implemented due to these provide an interactive and fairly straightforward operation, allowing users to be used comfortably. Moreover, Google Maps API works on the set of AJAX technologies, which have many advantages, such as using XML to exchange data over the network.

For the storage of images related to the flooding Flickr has been used because it allows upload, delete and share images. We could have chosen the option to save the images on the website that hosts the MAIZ application, and reference them from there to display when running the application, but, considering the need to store many images and videos, but it was decided to do this more dynamically, that is, display the picture but bringing it from a server dedicated to do that; even more, it allows to carry out through a cloud computing service in the future.

MAIZ also uses the Yahoo Weather Service for information about weather conditions in the relevant geographical area and its weather forecast. Therefore, Yahoo Weather is a source of data.

\subsection{Select development tools}

After analyzing and comparing the characteristics of two of the most popular tools for designing and creating websites (Dreamweaver and FrontPage), it was decided to perform the site using Dreamweaver because that it is more complete and suitable for this project.

To work and know the functional requirements, it was built database containing information of different kinds: river levels, neighborhood name, street name, data of emergency shelters, health centers, food pantry, etc. So that requires software that would do this, i.e., a Database Management System (DBMS).

MySQL is a DBMS that allows developing and effectively managing projects of this nature. This software is one of the most used, along with Oracle, to perform the data management computer systems.

It was decided to use MySQL as one of the DBMS software with more support for their use, these include: Heidi, Navicat and MyAdmin, even though this software is free and relatively easy to manipulate.

\subsection{Design, development and deploy}

The database developed consists of eight tables, enough to save the information necessary to take into account the objective of this project. Figure 3, which represents the entity relationship model of the database, clearly shows these eight entities.

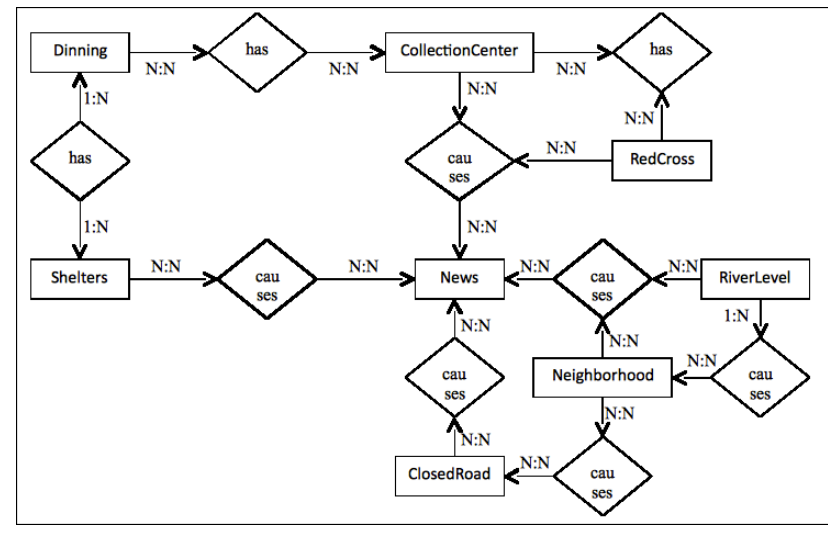

Fig. 3. Entity-Relationship model.

For data in the database application can be recognized by Google Maps API, this information must convert to XML format, so that can be viewed using any browser.

Google provides tools that allow developers to embed additions to their Websites quickly and easily. These are small applications called Web Elements that saves developers the task of programming complete modules due to users only need to configure the application so that it functions according to the needs. Someone can use Google elements as they are available for using on some blog or Website even for profit, as long as, it is authorized by Google.

Google Web elements can add content such as news, maps, calendar, translator and YouTube videos; also it is possible include social conversations Google Friend Connect. Google Web Elements are used very easily configured by choosing only the desired features, and copy the resulting code on the target website. These elements have a similar interface to the scheme of Google and not very customizable, but it is very easy to use and do not 
necessary be a professional developer to implement it on a website, but programmers can alter the resulting code.

MAIZ has considered News, Calendar, Conversation, and Custom Search Services.

The most important features of MAIZ is the RSS feed service. RSS is a syndication format for Web content that uses a dialect (language derived) of XML developed specifically for all types of sites that update information often and which it can share. This is known as Web retransmission or Web syndication.

Another Web service implemented in MAIZ is weather forecast. Yahoo Weather was used for it. Weather RSS feed enables to get up-to-date weather information for place. It is possible to save this feed in My Yahoo or feed aggregator, or incorporate the RSS data into website or client application. Weather RSS feed is a dynamically generated feed based on Yahoos WOEID (Where on Earth IDentifier). Feeds are provided free of charge for use by people and non-profit organizations for personal, noncommercial uses.

\section{MASHUP SERVICES}

The following describes the main features of MAIZ, both civil protection authorities and those people affected by flooding.

\subsection{Civil Protection Authorities' Services}

The main service offered by Mashup application to civil protection authority is the ability to capture and manage data that feed MAIZ information services to users. The authorities will be responsible for feeding the application with the data they obtain about river levels, flood affected neighborhoods and provide content RSS feed, to name a few. Figure 4 shows the capture screen through which the Mashup application administrators can record data about the affected neighborhood, among them is the identity, address and reference point. It can also to specify the path where is found an image that serves in order to allow users to explicitly identify the area refered.

As mentioned in section 4.4 (design and development) of the method for constructing Mashup applications, one features is the RSS Feed. Civil protection authorities using this service can utilize RSS Feed to disseminate important news to people affected by flooding. MAIZ managers are responsible for creating the content to be rebroadcast to users syndicated.

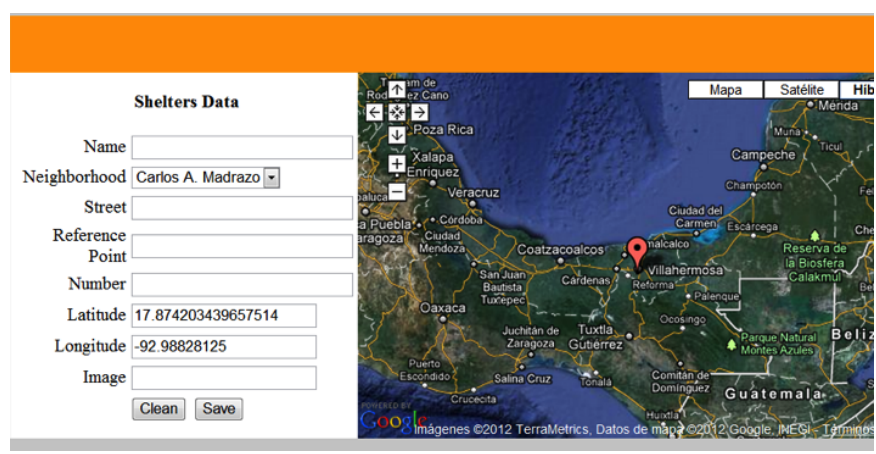

Fig. 4. Data capture screen shelters.

\subsection{Citizen's Services}

The Web Mashup application provides an integrated interface where users can get various information services. Figure 5 shows the application main menu.

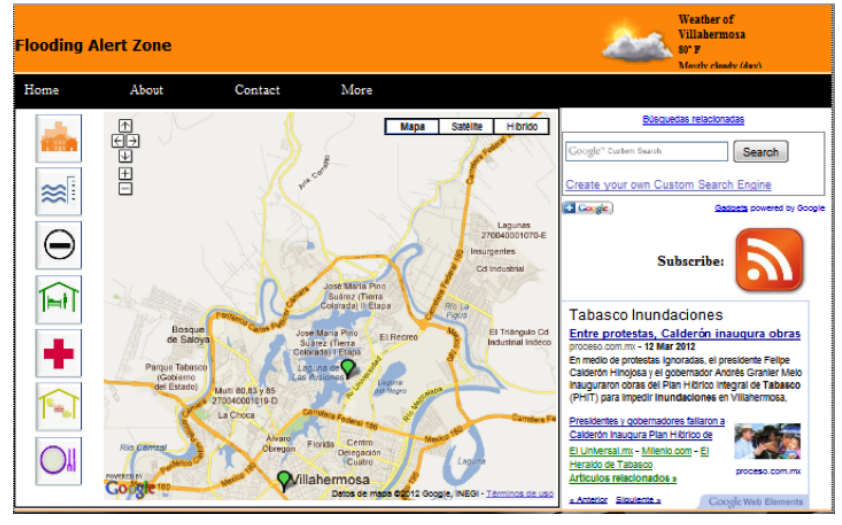

Fig. 5. Main Menu.

Table 1. MAIZ Information services

\begin{tabular}{|c|c|}
\hline $\begin{array}{l}\text { Color I } \\
\text { Icon }\end{array}$ & Functionality \\
\hline 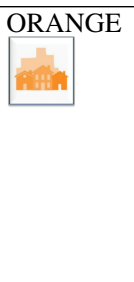 & $\begin{array}{l}\text { Neighborhood. This information service allows users } \\
\text { to know the geographic location of the neighborhoods } \\
\text { affected by floods. By clicking on this icon will ap- } \\
\text { pear "balloons" in the map of the city of Villaher- } \\
\text { mosa, indicating the affected neighborhoods, once } \\
\text { you click at a specific balloon can obtain more de- } \\
\text { tailed neighborhoods information. This is shown in } \\
\text { Figure } 6\end{array}$ \\
\hline BLUE & $\begin{array}{l}\text { River level. Service that allows to know rivers level } \\
\text { related to its critical scale }\end{array}$ \\
\hline BLACK & $\begin{array}{l}\text { Closed road. By using this icon shows the user the } \\
\text { paths or roads that are closed to traffic because they } \\
\text { are affected by floods }\end{array}$ \\
\hline GREEN & $\begin{array}{l}\text { Shelters. By using this service is obtained information } \\
\text { from the areas designated as emergency shelters for } \\
\text { those people affected. }\end{array}$ \\
\hline RED & $\begin{array}{l}\text { Red Cross (medical services and healthcare). It ob- } \\
\text { tains information (addresses) from centers offering } \\
\text { health services. Also Red Cross helps for emergen- } \\
\text { cies and keep people safe. }\end{array}$ \\
\hline YELLOW & $\begin{array}{l}\text { Collection center. Places where people receiving } \\
\text { goods, food or clothes that are donated by citizens or } \\
\text { civil services to mitigate the needs. }\end{array}$ \\
\hline PURPLE & $\begin{array}{l}\text { Food pantry (soup kitchen). Places where disaster vic- } \\
\text { tims can go and receive free foods. These are enabled } \\
\text { by civil protection authorities or NGOs. }\end{array}$ \\
\hline
\end{tabular}

Table 1 shows the service information obtained with the icons on the left side of the screen shown in Figure 5.

Fig. 6 shows that by clicking on a green balloon (emergency shelter) gives more detailed information, such as, users can get a list of people housed, the total capacity of the emergency shelter in the number of people and number of spaces available at that time. To consult the list of persons registered in the emergency shelter is extremely important for family members or friends of those housed are trying to find out an emergency shelter set up for how they are.

As can be noticed in icons column illustrated in Table 1, each service has a distinctive color. The aim of this classification is to differentiate each information service when these are georeferenced in a map of Villahermosa. Users can have in a single map different color balloons indicating places of various interest sites. 


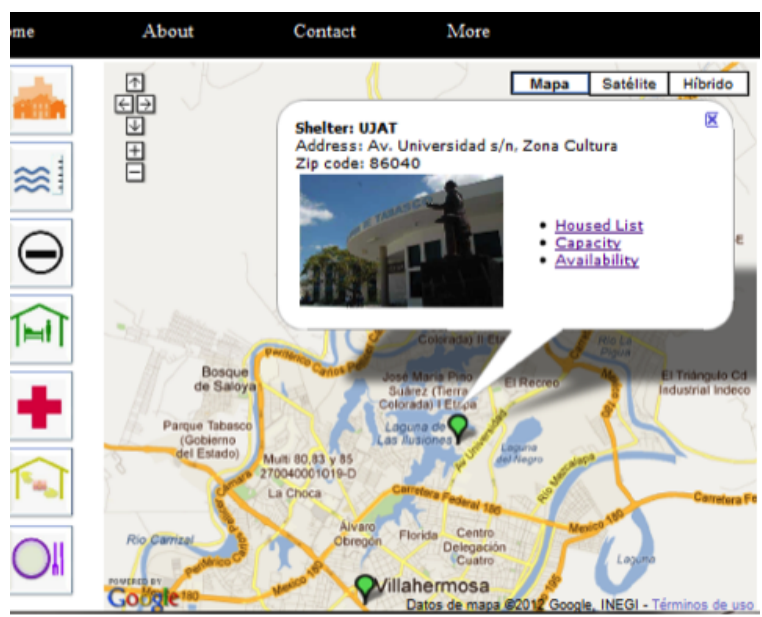

Fig. 6. Green ballon showing information, which indicate site for different services.

On the other hand, users must subscribe to the RSS service featured in MAIZ and through a RSS reader will be aware of important events during and after the contingency.

At the upper right corner of the screen is updated dynamically the weather for Villahermosa city. Shows the temperature in Celsius or Fahrenheit and environmental conditions (sunny, cloudy, rain, etc.).

The weather service, personalized search, RSS feed and news can be seen in Figure 7.

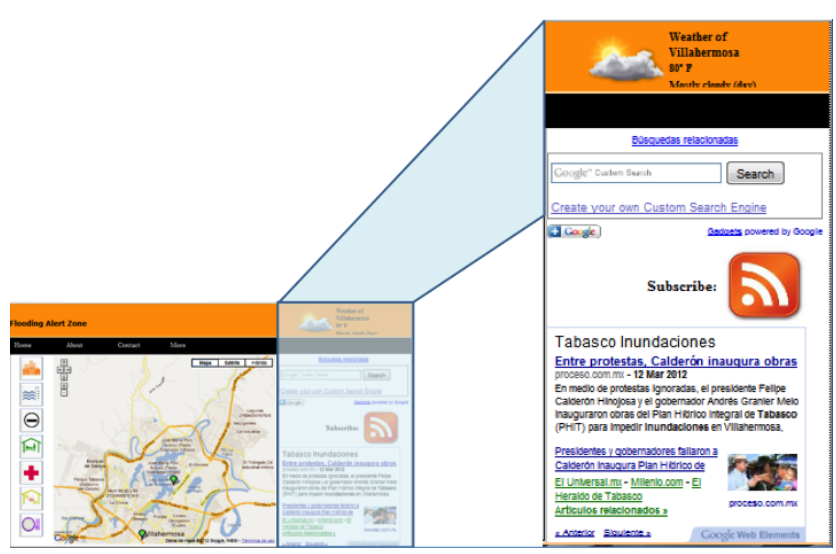

Fig. 7. Weather, personalized search and RSS feed Services.

\section{CONCLUSIONS}

Implementing various Web services within a Web application enables to create functional systems and added value. This allows to developers to mix and integrate Web services for providing to users with unique and different services. That is, make use of Web services that provide other sites or Web applications, thus having more complete applications in terms of functionality, without having personally developed the necessary code for it.

Mashup applications as part of Web 2.0, as shown in the case study, allows application users to get services and features difficult to aim if a third-party Web services is not possible. As shown in MAIZ, users can query maps to know geographic locations of point of interest (emergency shelters, health centers, food pantry, roads blocked) during a disaster situation, chat service (chat), custom searches, RSS feeds by which civil protection authorities make known them announcements of interest to the population, and so on.

Web Mashup application (MAIZ) developed meets the purpose of serving as a close liaison between civil protection authorities and people affected by floods, providing timely and valuable information for them.

\section{FUTURE WORKS}

It has depicted some ideas to build a user-centric intelligent environment, for instance, disaster victims being at their houses could launch a request for specific help (red cross, 066, bottled water, police, food, fire brigade, and so on) using a disaster chart or fridge magnet containing embedded images of services previously mentioned. Images printed on RFID tags are attached as sticker on the chart. Each image printed on RFID or QR code has information stored with users' identification and localization data. This information is labeled on RFID tags representing an emergency symbol. Using NFC technology on the mobile phone, users touch the icon, and the application sends an alert message via GPRS or $3 \mathrm{G}$ to rescuers. This application automatically sends users data previously stored on their mobile phones and their GPS coordinates, as well. Thus, users should not be typing or dragging or pushing buttons on the device. In order to achieve this, it is necessary that users wear mobile phone equipped with a radio-frequency reader and get closer for obtaining services like localization and personal information.

\section{Acknowledgement}

This work has been funded by the PROMEP - UJATABEXB182, POA 20120274. The authors wish to thank Emmanuel Vidal for programming the prototype. We are thanked for his support.

\section{REFERENCES}

[1] A. Kwasinski, W.W. Weaver, P.L. Chapman, and P.T. Krein. Telecommunications power plant damage assessment for hurricane katrina- site survey and follow-up results. Systems Journal, IEEE, 3(3):277 -287, sept. 2009.

[2] Banipal Kulwinder. Strategic approach to disaster management: lessons learned from hurricane katrina. Disaster Prevention and Management, 15(3):484 -494, 2006.

[3] Yingjie Yang, Xiaowen Kang, and Xin Du. Hierarchical disaster tolerant architecture based on virtual storage technology. In Networking and Digital Society, 2009. ICNDS 09. International Conference on, volume 2, pages 170 173, may 2009.

[4] Liangping Zhang and Weixing Wang. Constructions on disaster tolerant backup system of management information system. In Computer Science Education (ICCSE), 2011 6th International Conference on, pages 425 -427, aug. 2011.

[5] Shi Li, Zeng Ming, and Li Lingyun. A novel electricity marketing model integrating intelligent disaster-recovery system. Systems Engineering Procedia, 4(0):133 - 142, 2012. ice:title $i$ Information Engineering and Complexity Science - Part II.

[6] Miguel A. Wister, Pablo Pancardo, Francisco D. Acosta, and Dante Arias-Torres. Performance Evaluation of AODV and DYMO as a Plattform for Rescue Task Applications in MANETs. In Advanced Information Networking and Applications (WAINA), 2011 IEEE Workshops of International Conference on, pages 670-675, March 2011.

[7] Miguel A. Wister, Pablo Pancardo, and Francisco D. Acosta. Simulation-Based Analysis of Wireless Network 
for Disaster Information System. In UCAmI 2011 - 5th International Symposium on Ubiquitous Computing and Ambient Intelligence, December 2011.

[8] Wei Kang and Yoshitaka Shibata. Performance Evaluation of Disaster Information System Based on P2P network. In Advanced Information Networking and Applications Workshops (WAINA), 2010 IEEE 24th International Conference on, pages 710-715, 20-23 2010.

[9] J.C. Kim, D.Y. Kim, S.M. Jung, M.H. Lee, K.S. Kim, C.K. Lee, J.Y. Nah, S.H. Lee, J.H. Kim, W.J. Choi, and S.K. Yoo Implementation and Performance Evaluation of Mobile Ad Hoc network for Emergency Telemedicine System in Disaster Areas. In Engineering in Medicine and Biology Society, 2009. EMBC 2009. Annual International Conference of the IEEE, pages 1663 -1666, 3-6 2009.

[10] Hung-Chin Jang, Yao-Nan Lien, and Tzu-Chieh Tsai. Rescue information system for earthquake disasters based on manet emergency communication platform. In IWCMC '09: Proceedings of the 2009 International Conference on Wireless Communications and Mobile Computing, pages 623-627, New York, NY, USA, 2009. ACM.

[11] Suman Saha and Mitsuji Matsumoto. A Framework for Data Collection and Wireless Sensor Network Protocol for
Disaster Management. In Proceedings of the Second International Conference on COMmunication System softWAre and MiddlewaRE (COMSWARE 2007) Bangalore, India, pages 7-12. IEEE, January 2007.

[12] Yoshitaka Shibata, Yosuke Sato, Naoki Ogasawara, and Go Chiba. A Disaster Information System by Ballooned Wireless Adhoc Network. Complex, Intelligent and Software Intensive Systems, International Conference, 0:299_ 304, 2009.

[13] ALERT. ALERT USER GROUP system organization. http://www.alertsystems.org, 2012.

[14] Maneesha Sudhneer. Wireless Sensor Network for Disaster Monitoring. Wireless Sensor Networks: ApplicationCentric Design. In Tech. Janeza Trdine 9, 51000 Rijeka, Croatia, December 2010

[15] Val Jones, Georgios Karagiannis, and Sonia Heemstra de Groot. Ad hoc networking and ambient intelligence to support future disaster response. In H. Afifi and D. Zeghlache, editors, Proceedings of the 5th Workshop on Applications and Services in Wireless Networks (ASWNO5), pages 137-146. IEEE Communications Society, June 2005. 